\title{
An Innovator Nonintrusive Method for Disaggregating and Identifying Two Simultaneous Household Loads
}

\author{
Máximo Pérez-Romero ${ }^{1,2}$, Enrique Romero-Cadaval ${ }^{1}$, Adolfo Lozano-Tello $^{2}$, \\ João Martins ${ }^{3}$, and Rui Lopes ${ }^{3}$ \\ ${ }^{1}$ Power Electrical \& Electronics Systems R+D+i Group \\ University of Extremadura, Escuela de Ingenierías Industriales, Avda. de Elvas, s/n \\ 06006 Badajoz, Spain \\ ${ }^{2}$ Quercus Software Engineering Group \\ University of Extremadura, Escuela Politécnica, Avda. Universidad, s/n \\ 10071 Cáceres, Spain \\ ${ }^{3}$ Universidade Nova de Lisboa-FCT-DEE and UNINOVA-CTS \\ P-2829-516 Monte de Caparica, Portugal \\ mperez@peandes.net, \{alozano, eromero\}@unex.es, \\ jf.martins@fct.unl.pt, rm.lopes@campus.fct.unl.pt
}

\begin{abstract}
At the present time the monitoring systems are important in some areas, such as electric power supply industry and household environment because they provide useful information to energy storage and management tasks. A new nonintrusive monitoring method is proposed in this paper and it is able to disaggregate and identify two loads working simultaneously using a single measuring sensor and a least squares regression algorithm based on discrete form of the S-Transform.
\end{abstract}

Keywords: Monitoring, nonintrusive, disaggregation, identifying, simultaneously, S-Transform.

\section{Introduction}

The energy efficiency status report [1] showed by Joint Research Centre, or JRC, points out electricity consumption and efficiency trends in the EU-27 in 2012. According to the consumption statistics, final energy consumption in the EU-27 residential sector increased by $1.69 \%$, furthermore the lowest consumption level of the last 20 years was reached in 2007. In terms of final residential energy consumption means $26,65 \%$ of total final energy consumption in the year 2010 showed in the breakdown of the Fig. 1. This sector was the second most consuming part of total consumption, so the residential field is important in energy efficiency programmes and policies.

Smart grid concept is a communication infrastructure to connect different distributed energy resources with three main targets, that is, by adapting the demand side to the distributed energy sources, transforming the distributed energy resources into systems providing active energy and supporting services, such as reactive power supply, to stabilize the distributed network and using storage devices in demand 
side [2]. Smart grids enable a two-way exchange of information and power between producers and consumers, and this leads to increase transparency and promote responsible energy saving measures on the consumer's side. It is important that consumers are involved to obtain effective smart electricity systems through trust, understanding and clear tangible benefits and consumers become proactive consumers or prosumers, being necessary to know individual loads on demand side.

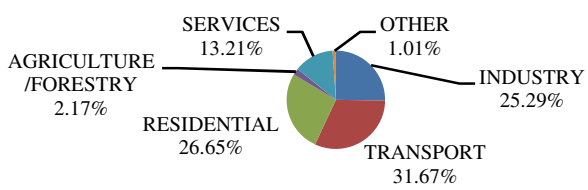

Fig. 1. Final energy consumption breakdown into sectors in the EU-27, 2010 (Eurostat)

Load monitoring systems are important to manage smart grids because they provide accurate power and energy usage information to power usage prediction and management. There are two usual methods in literature to monitor appliances: intrusive and nonintrusive methods.

Intrusive methods use sensors in each appliance to measure electric signals, such as voltage and current waveform. This measuring manner is expensive because installation needs time and one sensor for each appliance. For overcoming this drawback researchers have studied other procedure to extract the individual energy information of each device. It is called Nonintrusive Load Monitoring (NILM) or Nonintrusive Appliance Load Monitoring (NIALM) and it consist of a single sensor placed in the main breaker level to measure current and voltage and an algorithm for disaggregate and identify each one of appliance. This question was firstly studied by George W. Hart [3] of Massachusetts Institute of Technology (MIT) and published in the 90's.

The methods for disaggregating different appliances can be divided into two groups: the steady state analysis and the transient state analysis. Regarding first group, it is based on the change in steady state power and requires low sampling rates. Hart's work was already mentioned at the end of the previous paragraph. Next, Cole y Albicki [4] have proposed an extension to the Hart method. It uses edges and slopes as appliance features together with steady-state power draw. Finally, Baranski and Voss have developed an approach [5], [6], [7] for their system consisting of a histogram made up by historical data. As far as transient state analysis is concerned, transient characteristics can be used to disaggregate different household devices because they are tied to type of a given appliance. One of the most important researchs belongs to Leeb [8], [9]. An extension of work mentioned above consists of investigating the line voltage transients appeared when appliances are switched on or off (Cox and Leeb, [10]). Finally Chang proposed a transient energy detector [11].

A new method for disaggregating and identifying different household loads based on the S-transform [12] is proposed in this paper (other S-transform method was used by [13]). This work is part of [14]. The novelty is the use of mathematical regression along with the S-transform and the development of algorithm to be implemented with the software LabVIEW 2012. 


\section{Relationship to Collective Awareness Systems}

The Collective Awareness Platforms are ICT systems taking advantage of distributed knowledge and data from real environments, that is, Internet of things, to generate open databases. These types of platforms are scalable resulting in a big data source for different applications, such as real-time household power consumption data for managing energy demand of consumers, becoming collective tools.

In this way, this paper is based on such topic by disaggregating and identifying loads working individually or simultaneously and by sending such kind information in real-time to a decision central system.

\section{S-Transform}

The S-transform is a time-frequency analysis and representation technique proposed by Stockwell, Manshinha and Lowe in 1996. It is consists of a moving and scalable localizing Gaussian window leading to a time-frequency representation. Phase information is absolute because the origin of the time axis is taken as the fixed reference point.

The analysis begins with the target signal $h(t) . W(\tau, f)$ is the Continuous Wavelet transform (CWT) of the signal using a specific mother wavelet and its equation appears in (1).

$$
W(\tau, f)=\int_{-\infty}^{\infty} h(t) w(t-\tau, f) d t
$$

where $t$ is the time, $f$ is the frequency and $w(t-\tau, f)$ is the specific mother wavelet. Then

$$
S(\tau, f)=W(\tau, f) e^{-i 2 \pi f \tau}=\int_{-\infty}^{\infty} h(t) w(t-\tau, f) e^{-i 2 \pi f \tau} d t
$$

The mother wavelet is the first expression of (3).

$$
w(\tau, f)=g(t) e^{-i 2 \pi f t} ; g(t)=\frac{1}{\sigma \sqrt{2 \pi}} e^{-t^{2} / 2 \sigma^{2}} ; \sigma(f)=T=\frac{\lambda}{|f|} \quad \lambda>0 .
$$

The formula $g(t)$ is a Gaussian window and the parameter $\sigma$ means the Gaussian window width, where $\lambda$ sets frequency resolution. In this paper the parameter $\lambda$ has the value 1 . As the window width is the inverse of frequency, S-Transform results in a multiresolution analysis of the signal. Therefore, a high value of frequency gives high resolution of time and a low value of frequency gives high resolution of frequency.

Finally, by combining the expressions (4) and (2) the complete definition of the continuous S-Transform is given by (5).

$$
\begin{aligned}
& w(t-\tau, f)=\frac{|f|}{\sqrt{2 \pi}} e^{-(t-\tau)^{2} f^{2} / 2} e^{-i 2 \pi f(t-\tau)} \\
& S(\tau, f)=\int_{-\infty}^{\infty} h(t) \frac{|f|}{\sqrt{2 \pi}} e^{-(t-\tau)^{2} f^{2} / 2} e^{-i 2 \pi f(t-\tau)} e^{-i 2 \pi f \tau} d t .
\end{aligned}
$$


To efficiently implement on a computer the S-Transform it is necessary to develop the discrete form of the S-Transform. Stockwell et al. rewrote the continuous STransform by using Fourier transform $H(\alpha)$ of time series $h(t)$ as the equation (6) shows to get the discrete form. So, S-Transform can be run as computer algorithm by using the computational efficiency of Fast Fourier Transform and Inverse Fourier Transform in (6).

From the discrete time series $h[k T]$ corresponding to $h(t)$ with a sample time $T$ the equation of the discrete Fourier transform is given by (7).

$$
\begin{array}{ll}
S(\tau, f)=\int_{-\infty}^{\infty} H(\alpha+f) e^{-\left(2 \pi^{2} \lambda^{2} \alpha^{2}\right) / f^{2}} e^{i 2 \pi \alpha \tau} d \alpha & f \neq 0 . \\
H\left[\frac{n}{N T}\right]=\frac{1}{N} \sum_{k=0}^{N-1} h[k T] e^{-i 2 \pi n k / N} & n=0,1, \ldots, N-1 .
\end{array}
$$

where $k$ and $n=0,1, \ldots, N-1$.

Therefore, the discrete S-Transform is gotten by setting $f \rightarrow n /(N T)$ and $\tau \rightarrow j T$ resulting in (8) and (9), with $\lambda$ equal to 1 .

$$
\begin{aligned}
& S\left[j T, \frac{n}{N T}\right]=\sum_{m=0}^{N-1} H\left[\frac{m+n}{N T}\right] e^{-2 \pi^{2} m^{2} / n^{2}} e^{i 2 \pi m j / N} \quad n \neq 0 . \\
& S[j T, 0]=\frac{1}{N} \sum_{m=0}^{N-1} h\left(\frac{m}{N T}\right) \quad n=0 .
\end{aligned}
$$

where $j, m$ and $n=0,1, \ldots, N-1$.

The S-Transform consists of a complex matrix (called S-matrix) with dimension $\mathrm{N}$ by $\mathrm{M}$ where rows correspond to frequency and columns correspond to time.

\section{Case Study}

The proposed system consists of parts showed in Fig. 2: a monitor, a light bulb, a single current sensor of the measuring board, a point of common coupling (PCC), a control platform sbRIO 9631 of National Instruments consisting of a FPGA (hardware part) and a real-time processor (software part) and a notebook being only used to program the control platform. Overall system communicates with the central server called CEMIS.

The current sensor measures the current demanded by complete system and its information is sent to analog input of the control platform for disaggregating and identifying what loads are switched on or switched off. The two loads are connected in parallel to the PCC and one or both of them can be working simultaneously. The programming methodology of disaggregation and identification tasks, main topic of this paper, is based on codesign hardware-software and developed in LabVIEW 2012. The FPGA will get data from current sensor because the analog input is connected to it directly. It will also perform preprocessing tasks such as scale current data, convert from data type of fixed-point to unsigned word of 32 bits and write to a memory buffer. The sample frequency is $10 \mathrm{kHz}$ and the number of points $\mathrm{N}$ is 201 . 


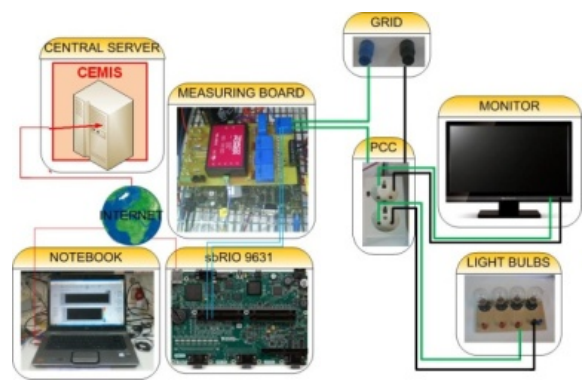

Fig. 2. System diagram

Fig. 3(a) shows the FPGA algorithm flowchart. The software part will read the data from buffer, convert from unsigned word of 32 bits to data type of double precision, extract one signal cycle, execute the S-transform and disaggregate and identify the loads by means of least squares regression as it is shown in the flowchart of Fig. 3(b).

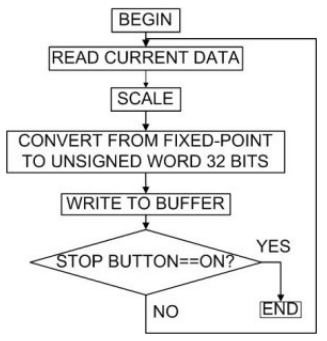

(a)

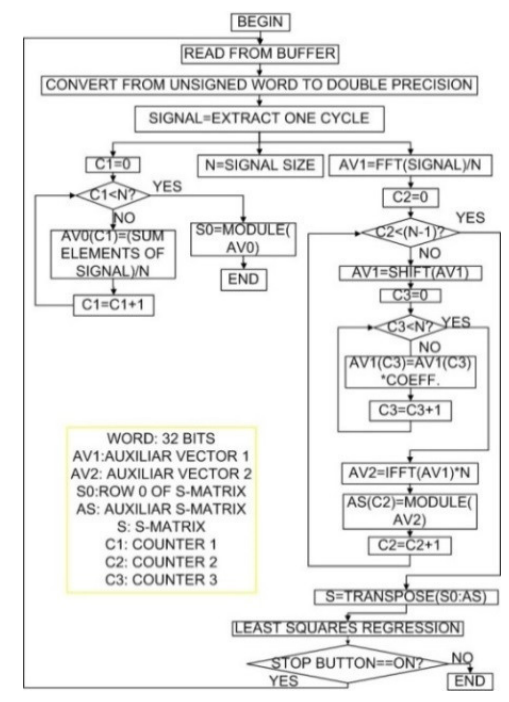

(b)

Fig. 3. Flowcharts: (a) FPGA algorithm, (b) Processor algorithm

The resultant matrix " $\mathrm{S}$ " is a transposed complex matrix with $\mathrm{N}$ rows and $\mathrm{M}=$ $\mathrm{N} / 2+1$ columns, that is, $201 \times 102$. The task of identification consists of the least squares regression (10).

$$
\sum_{i=1}^{N}\left(z_{i}-\left(a_{1} x_{i}+a_{2} y_{i}\right)\right)^{2}=e .
$$

where $Z_{i}$ is the $S$-Transform of the overall measured current signal, $x_{i}$ is the S-Transform of the pattern signal of linear load (light bulb), Fig. 4(a), $y_{i}$ is the 
S-Transform of the pattern signal of electronic load (monitor), Fig. 4(b), $a_{1}$ and $a_{2}$ are the amplitudes of such pattern signals, respectively, and $e$ is the least squares error. The coefficients $a_{1}$ and $a_{2}$ are obtained in (12) from (11) and they can be used in the time domain because the $\mathrm{S}$-Transform meets the linearity criteria.

$$
\begin{aligned}
& \frac{\partial e}{\partial a_{1}}=\sum_{i=1}^{N} 2\left(z_{i}-a_{1} x_{i}-a_{2} y_{i}\right) x_{i}=0 ; \frac{\partial e}{\partial a_{2}}=\sum_{i=1}^{N} 2\left(z_{i}-a_{1} x_{i}-a_{2} y_{i}\right) y_{i}=0 . \\
& a_{1}=\frac{B D-C E}{A B-C^{2}} ; a_{2}=\frac{A E-C D}{A B-C^{2}} ; A=\sum_{i=1}^{N} x_{i}^{2} ; B=\sum_{i=1}^{N} y_{i}^{2} ; C=\sum_{i=1}^{N} x_{i} y_{i} ; D=\sum_{i=1}^{N} z_{i} x_{i} ; E=\sum_{i=1}^{N} z_{i} y_{i} .
\end{aligned}
$$

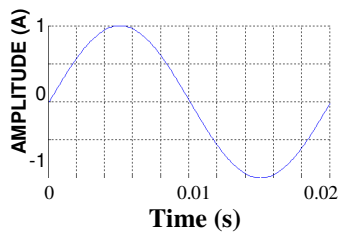

(a)

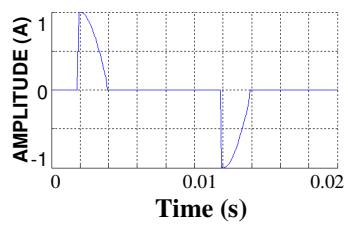

(b)

Fig. 4. (a) Current signal of light bulb. (b) Current signal of monitor.

\section{$5 \quad$ Experimental Results}

On one side, for the case where a single light bulb is switched on the Fig. 5(a) shows signal fitted and computed by the system algorithm (white colour) and measured current signal of light bulb (yellow colour) and Fig. 5(b) shows the S-Transform of measured signal. The coefficients $\mathrm{a}_{1}$ and $\mathrm{a}_{2}$ have values 0,368337 and 0,0163964 (Table 1), respectively, and identify a linear load as white colour signal of the Fig. 5(a) illustrates.

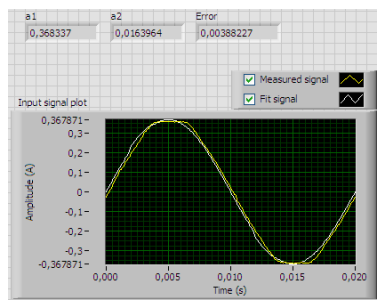

(a)

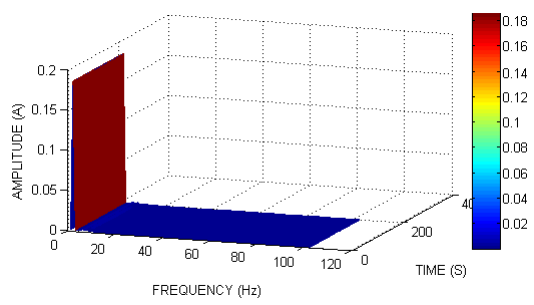

(b)

Fig. 5. (a) Measured and fitted current signal of light bulb (front panel of LabVIEW 2012). (b) S-Transform of measured current signal.

On the other hand, when a single electronic load or monitor is turned on the system discovers a nonlinear load currently working. The coefficients are shown in Table 1 (this table illustrates the coefficients and error for each appliance and the combination of both.) and its waveform appears in Fig. 6(a) whereas its S-Transform is shown by Fig. 6(b). Finally, the main case consists of one light bulb and monitor working 
simultaneously. The system calculates the values of coefficients $a_{1}$ and $a_{2}$ and, according to the gotten results, it can be said that two loads have been disaggregated and identified. Fig. 7(a) shows time series signals and Fig. 7(b) presents the S-Transform.

Table 1. Values of coefficients

\begin{tabular}{llll}
\hline LOAD & $\mathrm{a}_{1}$ & $\mathrm{a}_{2}$ & Error \\
\hline Light bulb & 0,368337 & 0,0163964 & 0,003888227 \\
Monitor & 0,0450099 & 0,309192 & 0,220845 \\
Light bulb and monitor & 0,409668 & 0,321245 & 0,181646 \\
\hline
\end{tabular}

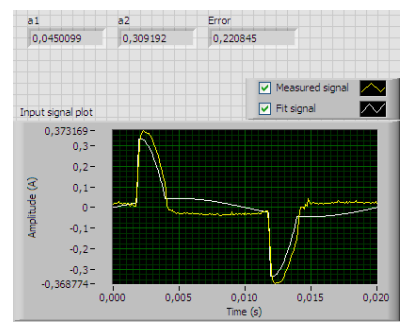

(a)

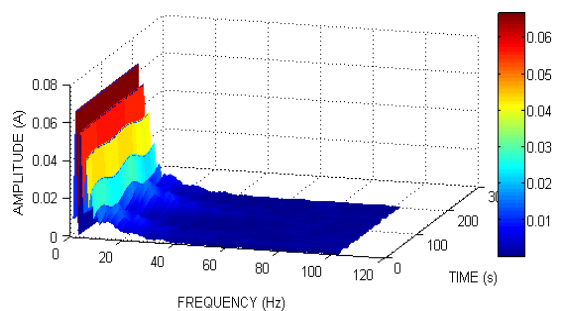

(b)

Fig. 6. (a) Measured and fitted current signal of monitor (front panel of LabVIEW 2012). (b) STransform of measured current signal.

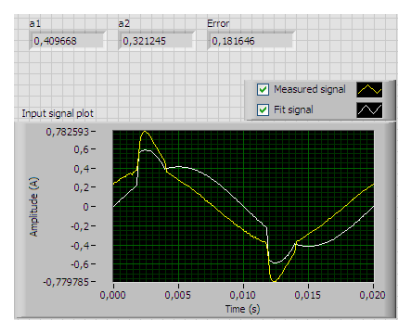

(a)

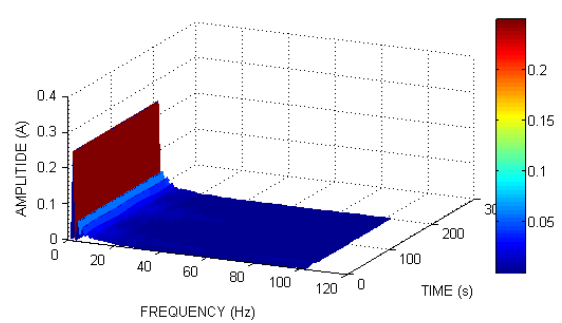

(b)

Fig. 7. (a) Measured and fitted current signal of light bulb and monitor (front panel of LabVIEW 2012). (b) S-Transform of measured current signals.

\section{Conclusion and Further Work}

The main feature of this new nonintrusive method is to disaggregate and identify two loads, a linear device and nonlinear device, by using S-Transform and least squares regression with one cycle of the time series signal. It is an advantage because the $S$ Transform uses computational efficiency of FFT and IFFT and other parameters are not necessary, such as harmonic analysis and reactive power, reducing computations. Furthermore, the system needs a single current sensor reducing cost. In the future, 
more loads will be connected to the system and the least squares regression will extend to detect such devices although the complexity will increase. There is also an open work to identify devices switched on by using neural networks.

Acknowledgements. This work has been developed under support of Telefónica Chair of University of Extremadura.

\section{References}

1. JRC. EESR (2012), http://iet.jrc.ec.europa.eu/energyefficiency/node/7250

2. Gregor, R., Simon, F., Ulf, J.J., Hahnel, P.B., Bernhard, W.-H.: What the term Agent stands for in the Smart Grid Definition of Agents and Multi-Agent Systems from an Engineer's Perspective. In: Proceedings of the Federated Conference on Computer Science and Information Systems, Wroclaw, Poland, pp. 1301-1305 (2012)

3. Hart, G.W.: Nonintrusive Appliance Load Monitoring. Proceedings of the IEEE, 1870-1891 (1992)

4. Cole, A., Albicki, A.: Algorithm for nonintrusive identification of residential appliances. In: Proceedings of the 1998 IEEE ISCAS, pp. 338-341 (1998)

5. Baranski, M., Voss, J.: Non-Intrusive Appliance Load Monitoring Based on an Optical Sensor. In: EEE Power Tech Conference, Bologna (2003)

6. Baranski, M., Voss, J.: Genetic Algorithm for Pattern Detection in NIALM Systems. In: IEEE International Conference on Systems, Man and Cybernetics, pp. 3462-3468 (2004)

7. Baranski, M., Voss, J.: Detecting Patterns of Appliances from Total Load Data Using a Dynamic Programming Approach. In: Fourth IEEE International Conference on Data Mining, ICDM 2004 (2004)

8. Leeb, S.B., Kirtley, J.L., Levan, M.S., Sweeney, J.P.: Development and Validation of a Transient Event Detector. AMP J. of Technology, 69-74 (1993)

9. Leeb, S.B., Shaw, S.R., Kirtley, J.L.: Transient Event Detection in Spectral Envelope Estimates. Power, 1200-1210 (1995)

10. Cox, R., Leeb, S.B., Shaw, S.R., Norford, L.K.: Transient Event Detection for Nonintrusive Load Monitoring and Demand Side Management Using Voltage Distortion. Computer Engineering, 1751-1757 (2006)

11. Chang, H.-H., Yang, H.-T., Lin, C.-L.: Load Identification in Neural Networks for a Nonintrusive Monitoring of Industrial Electrical Loads. In: Shen, W., Yong, J., Yang, Y., Barthès, J.-P.A., Luo, J. (eds.) CSCWD 2007. LNCS, vol. 5236, pp. 664-674. Springer, Heidelberg (2008)

12. Stockwell, R.G., Mansinha, L., Lowe, R.P.: Localization of the complex spectrum: the S transform. IEEE Trans. Signal Processing, 998-1001 (1996)

13. Martins, J.F., Lopes, R., Lima, C., Romero-Cadaval, E., Vinnikov, D.: A Novel Nonintrusive Load Monitoring System Based on the S-Transform. In: 13th International Conference on Optimization of Electrical and Electronic Equipment (OPTIM 2012), Brasov, Romania, May 24-26 (2012)

14. Pérez-Romero, M., Gallardo-Lozano, J., Romero-Cadaval, E., Lozano-Tello, A.: Local Energy Management Unit for Residential Applications. Electronics and Electrical Engineering 19(7), 61-64 (2013) 